\title{
Measurement and assessment method for ship radiating transient noise
}

\author{
YU Tong-kui ${ }^{1,2, a}$, LIU Wen-shuai ${ }^{2, b}$, Shi Sheng-guo ${ }^{1, c}$ and Long Jun ${ }^{2, d}$ \\ ${ }^{1}$ College of Underwater Acoustic Engineering Harbin Engineering University, Harbin 115000, China \\ ${ }^{2}$ Dalian Scientific Test and Control Technology Institute, Dalian 116013, China \\ a ytk2222@163.com, bshishengguo@hrbeu.edu.cn, ${ }^{c}$ ytkxyz@163.com, ${ }^{d}$ longjun3159@163.com
}

Keywords: transient noise; kurtosis statistics; assessment method

\begin{abstract}
To solve the assessment problem of underwater radiated noise under the typical transient condition for ship, time-frequency analysis, FIR filter and high-order static are combined based on the energy computation method of transient signal, and the advantage of each method are utilized to obtain the total energy and the characteristic frequency spectrum of transient noise. The comparison platform of transient noise under different conditions is established, so the assessment of ship radiated transient noise can be realized. The experiment is developed under the condition of anchoring at the sea, and the impulse signal and amplitude modulated single frequency signal are both launched. The established assessment method of transient signal are used to analyze the received transient noise, 1/3oct frequency spectrum of transient noise and its energy at the frequency range of $20 \mathrm{~Hz} \sim 50 \mathrm{kHz}$ are calculated, the difference between the energy of total frequency and actual amount of energy is less than $1 \mathrm{~dB}$. As mentioned above, this method possesses the important engineering application value for the measurement and assessment of ship radiated transient noise.
\end{abstract}

\section{Introduction}

As we all know that warship radiation noise could be divided into two categories including steady noise and transient noise. Steady noise is consist of mechanical noise , hydrodynamic noise and propeller noise when the ship is voyaging on the sea in a steady condition. Line spectrum and continuous spectrum are the most conspicuous characters in the view of spectrum, Both of which could be the source of information of detectionand recognition by the sonars; Transient noise is formed by the launch process of weapons 、 the operation of rudder etc, with the characters of strong energy s short time of duration and frequently occurred times. As for the sonar,these characters may not just do favors for detecting but also provide important evidence for further recognition of warships. The acoustic characters of warships are the core of sonar detection and vibration noise reduction work.It is thus quite important to derive transient noise level and acoustic characters from the real estimation data.

There are many kinds of transient noise in ship, their duration and spectrum characteristics are different. In order to accurately evaluate these transient noise, we must establish a unified compare platform(Total energy and 1/3oct spectrum in the same frequency band). In order to achieve the goal, we first need to detect transient noise and obtain the generation and end time. In view of this problem, a lot of research work has been carried out at home and abroad, and many related detection are proposed, including narrow band[1],Power-law[2], partial instantaneous energy density[3], high-order static[4-5] and so on. Most of these detection methods are limited to the maximum peak value detection, and it is difficult to achieve generation and end time of transient noise. Because of these reasons, the method is proposed based on kurtosis ststistics and second order partial derivative function in this paper. In condition, aiming at the problem of transient noise level contrast, the method is proposed to obtain the total energy by integration of transient signal. The proposed method in this paper is proved to have good engineering application value by both theoretical simulation and experiments research. 


\section{Theoretical Analysis}

\subsection{Research on the transient signals energy calculation methods}

As for the transient signals and the conditions that the signals may distort during transmit process or encounter objects, it is better to use sound wave energy flux density to illustrate the signals. Energy flux density is the integration of transient sound intensity, that is:

$$
E=\int_{0}^{\infty} I d t=\frac{1}{\rho c} \int_{0}^{\infty} p^{2} d t
$$

Among which, $I$ is the transient sound intensity, $P$ is the transient sound pressure, $\rho c\left(\rho c=1.5 \times 10^{5} \mathrm{~g} / \mathrm{cm}^{2} . s\right.$ in the sea $)$ is the specific acoustic resistance.

Disturb noise is not needed when calculating energy out using integration in time-domain, presuming disturb noise is continuous, in that way can we take out the energy during a time interval T at a random selected time $T_{n}$, here is the specific formation:

$$
S=10 \log _{10}\left[\int_{0}^{T} p^{2}(t) d t-\int_{T_{n}}^{T_{n}+T} p_{n}{ }^{2}(t) d t\right]
$$

Among which $p(t)$ is the transient signal sound pressure, $p_{n}(t)$ is the disturb noise sound pressure.

\subsection{FIR filter based on windows function}

The aiming of design FIR filter is to confirm the coefficient $b$ on the basis of a given performance index, it could also be seen as the unit impulse sequence $h(n)$, which has got a limited length.

Presuming $H_{t}\left(e^{j \omega n}\right)$ is the ideal frequency response function which the standard demands, that is:

$$
H_{t}\left(e^{j \omega n}\right)=\sum_{n=\infty-}^{\infty} h_{t}(n) e^{-j \omega n}
$$

Among which $h_{t}(n)$ is the response sequence which is correspondent to the unit impulse.

\subsection{Transient noise judgment technology based on statistic}

Statistic analysis method is achieved on the basis of information derived from a number of samples. During the transient analysis process, which under the condition without sufficient quantity of samples nor the far more conspicuous time-varying characteristic, statistic analysis may still do a positive favor to a durable transient signal which could be viewed has got statistic characters within a short time,as for the signal component which can not meet the requirement of ergodic property,an obvious influence could be found in the output of kurtosis statistic detector because there does exist a kind of leap within the deployment which is changed relating to the leap of statistic characteristics. Resulting in that kurtosis of stable sequence analysis is regarded as statistic variables, and further discussion using kurtosis statistic variables could be done to make combined judgment.

During the detailed analysis of transient signals, a durable samples could be approximately regarded as alongside with statistic property respectively.Kurtosis could be estimated by fourth moment, the gathering characteristic of samples could be reflected by the size of second center moment, while the second center moment is the value of variance, which could reflect the level of gathering mean value nearby to some extent.However sometimes the deployment of the same variance may result in different flat even smooth levels, in order to symbolise the difference of the kurtosis levels within the deployment,the four center moment should be used.While as a matter of fact the real standard is the specific value of four center moment and $\sigma^{4}$, which could be called as kurtosis statistic, defined as:

$$
k=\frac{E\left\{[x-E(x)]^{4}\right\}}{\sigma^{4}}
$$


The formation above could be used to calculate the obtained test data, through which could reflect the variation of the data towards statistic deployment characteristics, resulting in the process to detect whether transient noise is included or not.

On the basis of the theories above, the advantage functions of all kinds of algorithms could be exerted comprehensively to form the methods to analysis transient noise data,the detailed diagram is showed as Fig1 below:

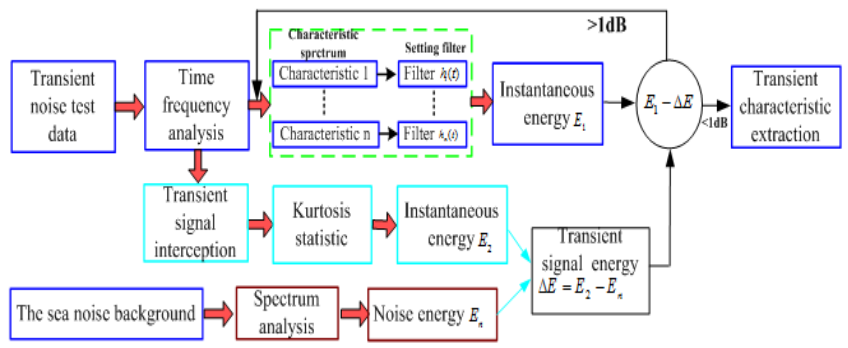

Fig1 Transient noise analysis process diagram

\section{Simulation}

Simulation

$$
\text { conditions: }
$$

exponent

signal

$x=g(t) e^{j \omega t}$

,modem

frequency $\omega=100 \mathrm{~Hz}, g(t)=e^{-\frac{t}{2} \sqrt{\frac{\pi}{N}}}$,superposition overlay Gaussian white noise ,all the initial phase position is SNR 6dB with the time interval 0.1s.

The simulation signal is the kind of product by frequency modulation on $100 \mathrm{~Hz}$ and amplitude signals, the characteristic frequency spectrum is not a single one,so the band pass frequency should be selected as wide as possible to secure no signal leakage.FIR filter of Hanning window is selected,the main lobe width is $8 \pi / N$,the band pass frequency dropped in $50 \mathrm{~Hz}-150 \mathrm{~Hz}$, the phase number is 100 ,the decrease is $-35 \mathrm{~dB}$ from the main lobe to the first side lope in consideration of band pass ripples of the filter.The diagrams of amplitude-frequency and phase frequency are as to Fig2,from which the phase frequency curve is liner phase strictly within the band pass,it could ensure the high fidelity after smoothing frequency.
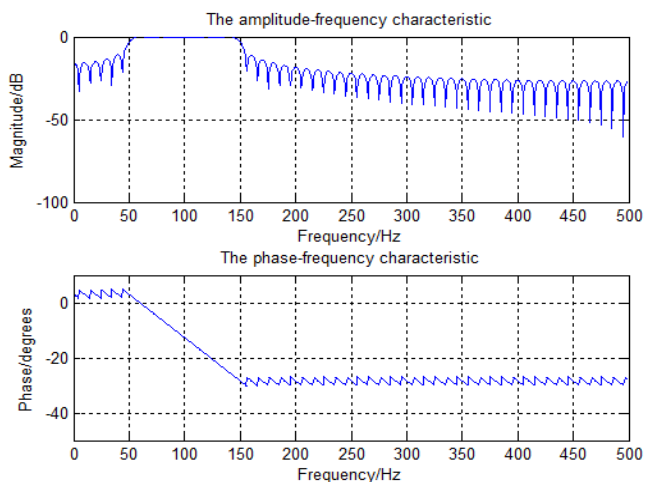

Fig2 Amplitude frequency and phase frequency response curve of the filter
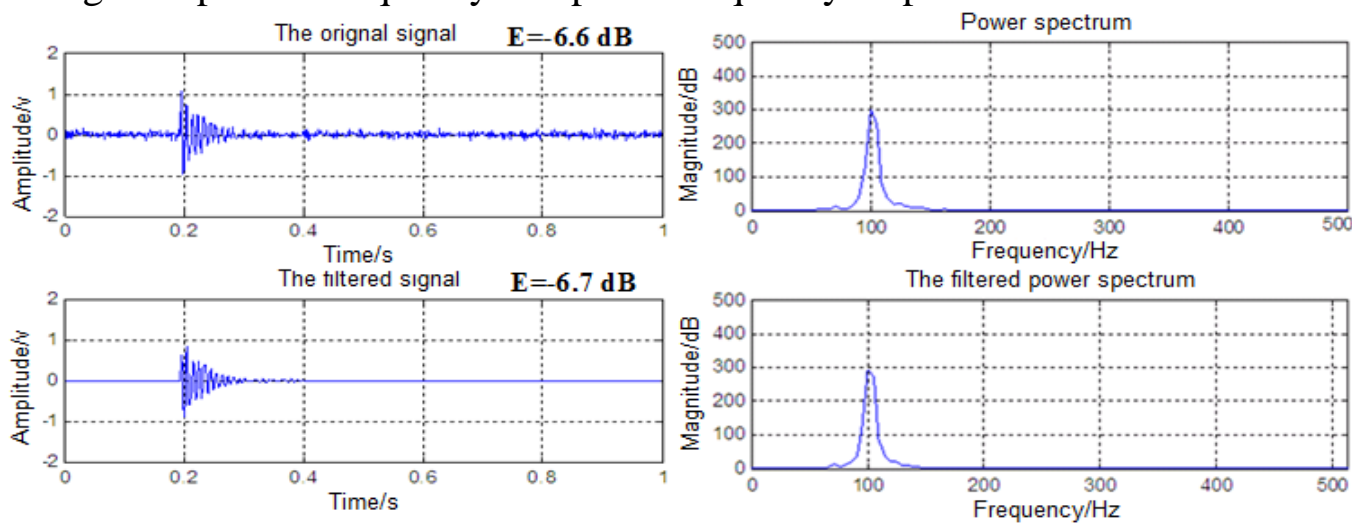

Fig3 The time-domain and frequency-domain wave form before and after filtering 
It could be inferred from Fig3 that no obvious change could be detected in comparison with the change before and after, the initial power of signal is $-6.6 \mathrm{~dB}$,the value becomes $-6.7 \mathrm{~dB}$ after filtering process. As comparison results shown, the difference is very minute. The source of the error mainly comes from the noise outside the filter frequency: The characteristics of frequency spectrum is totally the same through comparing the power spectrum before and after the filtering process.

As a whole, the characteristic frequency spectrum of transient signals and the magnitude of power could be accurately obtained through FIR filtering methods.

\section{Experimental Validation}

The experiments were achieved on the sea, the platform was a research ship, the transmit transducers and hydrophones were respectively installed on the water well's head and tail elastic stretched hanging bracket. The experiment of sound source transmitting and receiving process were operated on the flat sea.The known conditions were the sound source transmitting frequency, amplitude and phase, through which the radiation information in the water media could be obtained in the same time, the transient noise information processing method in the previous chapters could be used to analysis the data obtained,the real transmit signal could be compared simultaneously in order to test whether the algorithm is reliable or not.

The component of the system is shown as Fig4,mainly including transmitting system and receiving system,the transmitting system was consist of signal source 、 power amplifier and transmit transducer (UW350),receiving system was consist of hydrophones 、 signal masher 、 collector and laptop computers.

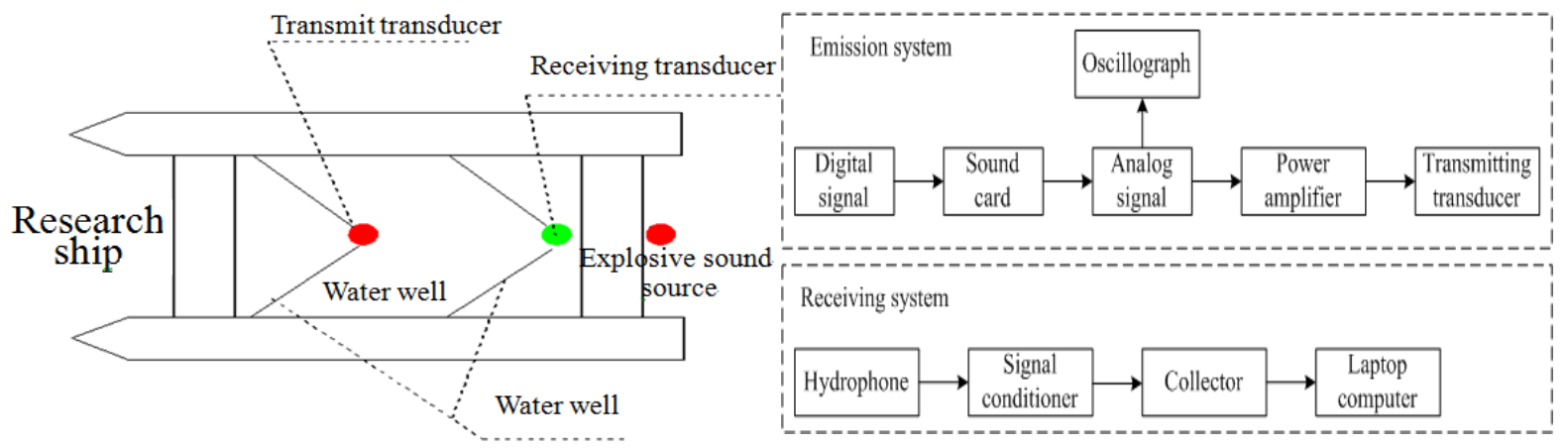

Fig4 The schematic diagram of test system and the component of experiment system

The working condition of the experiments were designed to simulate two typical non-steady types of signals:Impulse signal and amplitude modulation signal,the impulse signal was simulated by the explode sound source and the Gaussian modulation signal that amplitude 、 frequency and phase were known by the transducer,radiation noise sampling frequency was $100 \mathrm{kHz}$, the complete recording process was secured during the experiment.

The related job should be spread based on the data obtained and the transient noise analysis methods ,the detailed procedure was shown as Fig.5.

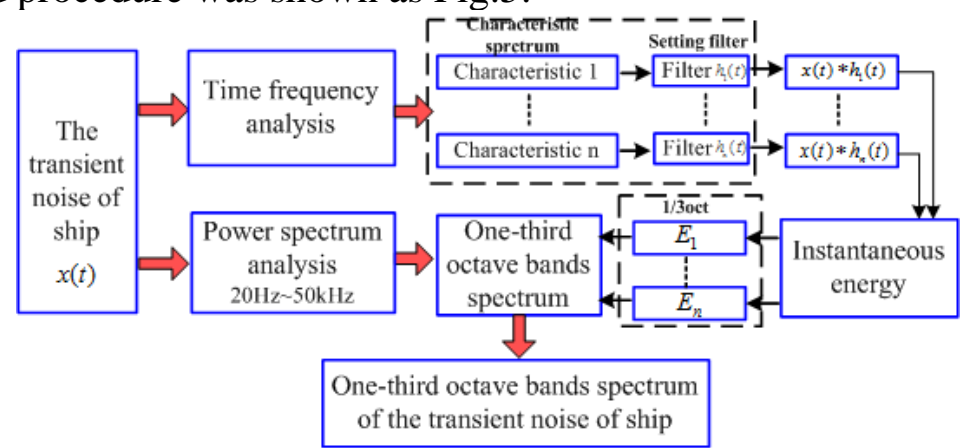

Fig5 The analysis process picture of sound source testify experiment data 
a. Explosive sound source working condition

Because the transient signal caused by explosive sound source duration time is too short, one second data obtained the characteristic signal was selected. The specific analysis is as follows.
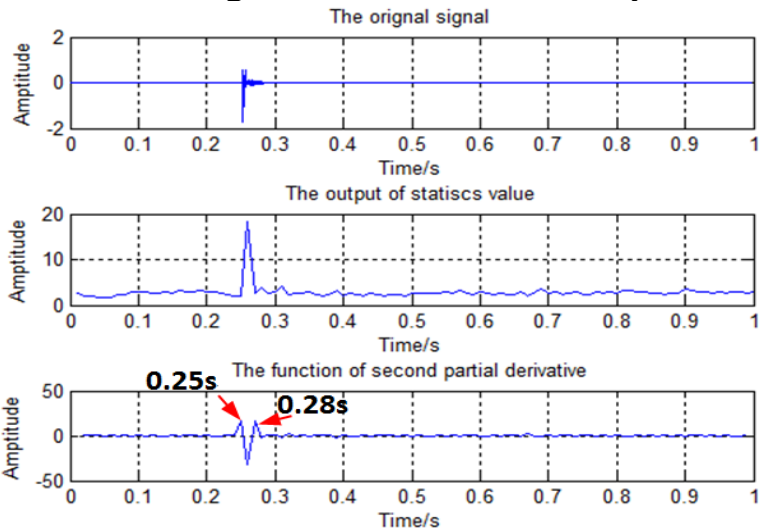

Fig6 The generation and end time of explosive sound source signal wave form

The time-domain waveform received during the explosive sound source was displayed by Fig6,which also includes the discriminating result at the generation and end time. Several points could be inferred from the fig including the signal's power which is pretty high and caused by explosive sound source,of course much higher than background noise,a very deep leading edge is performed,the duration time is short and belongs to a typical impulse type of signal,the generation and end time of the explosive signal also could be obtained in comparing the crest and trough of the waveform; The starting time was $0.25 \mathrm{~s}$ through the judgment of kurtosis statistical value,ending at $0.28 \mathrm{~s}$,duration time is $0.04 \mathrm{~s}$, which the time interval included the whole staring and ending time of the impulse signal.

According to the calculating method of the power using transient signal,the transient signal power of the explosive sound source working condition could be summarized as:

$$
S_{\text {all }}=10 \log _{10}\left[\int_{0}^{T} p^{2}(t) d t-\int_{T_{n}}^{T_{n}+T} p_{n}{ }^{2}(t) d t\right]+L M D+10 \lg D=176.8 d B
$$

Which $p(t)$ and $p_{n}(t)$ are respectively the noise sound pressure of transient signal and sea background, $L M D$ is the sensitivity of the hydrophone, $D$ is the distance between hydrophone and the explosive sound source.

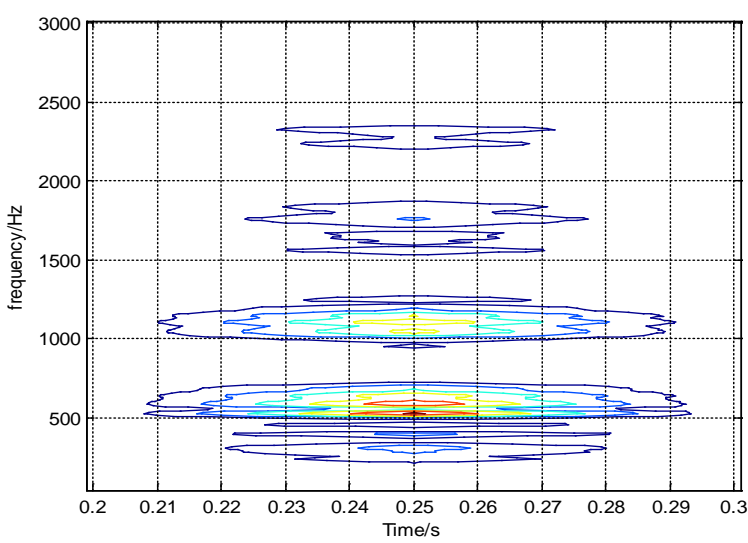

Fig7 The analysis result of the explosive sound source condition

The received analysis result of radiation noise time-frequency under the explosive noise working condition was given by Fig7,it could be inferred that frequency spectrum characteristic performs as a wide band,mainly stands in $213 \mathrm{~Hz} \sim 726 \mathrm{~Hz}$ 、 $939 \mathrm{~Hz} 1269 \mathrm{~Hz} 、 1531 \mathrm{~Hz} \sim 1873 \mathrm{~Hz}$ and $2197 \mathrm{~Hz} \sim 2349 \mathrm{~Hz}$, the amplitude of the power within these characteristic frequency range was given by procedures below. 

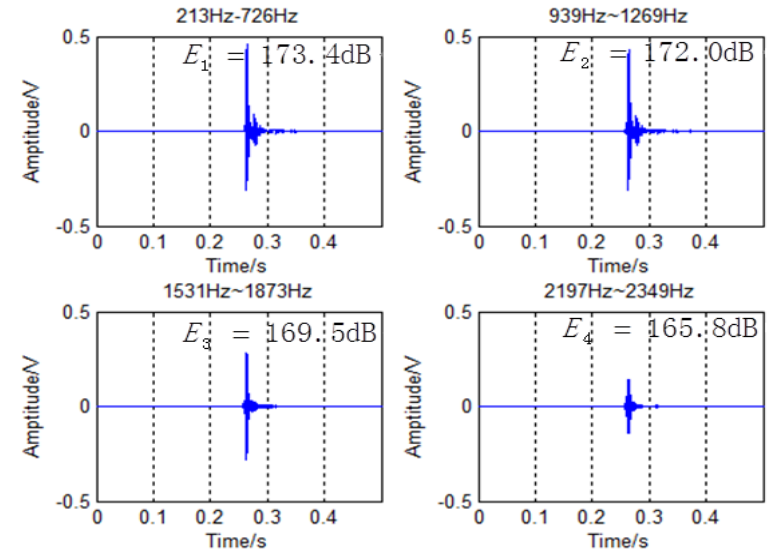

Fig8 The time-domain wave form and power of characteristic signal before and after filtering

The waveform and the amplitude of the power before and after filtering could be given by Fig8,inferring that the frequency band power within $213 \mathrm{~Hz} 726 \mathrm{~Hz} 、 939 \mathrm{~Hz} 1269 \mathrm{~Hz}$ 、 $1531 \mathrm{~Hz} \sim 1873 \mathrm{~Hz}$ and $2197 \mathrm{~Hz} \sim 2349 \mathrm{~Hz}$ is respectively $173.4 \mathrm{~dB} 、 172.0 \mathrm{~dB} 、 169.5 \mathrm{~dB}$ and $165.8 \mathrm{~dB}$,the added value of power is $177.4 \mathrm{~dB}$, the power difference is $0.7 \mathrm{~dB}$ compared to the initial transient signal,which proved the accuracy of the derivation of the transient signal.

On the basis of the analysis above,the $1 / 3$ oct sound pressure spectrum level was got through replacing $1 / 3$ oct frequency spectrum power in relating to the characteristic frequency band,as for what Fig9 displayed,the explosive sound source main working frequency band stands in $160 \mathrm{~Hz} 3150 \mathrm{~Hz}$,in which $800 \mathrm{~Hz}$ performed as a "concave" shape,because the transmit frequency of the explosive sound was too low, the validity of the analysis result from another side was thus proved.

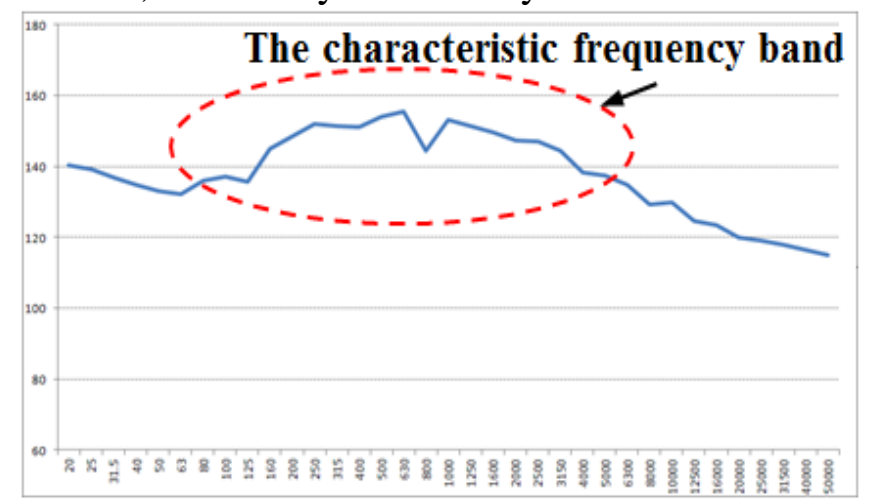

Fig9 1/3 sound pressure spectrum source level of radiation noise

b. Gaussian amplitude modulation signal working condition

A kind of Gaussian signal which stands at $6000 \mathrm{~Hz}$ was transmitted by the acoustic transducer,the transmitted signal was obtained by the hydrophone,here is the analysis referring to the obtained radiation noise data, and the detailed analysis result is below.
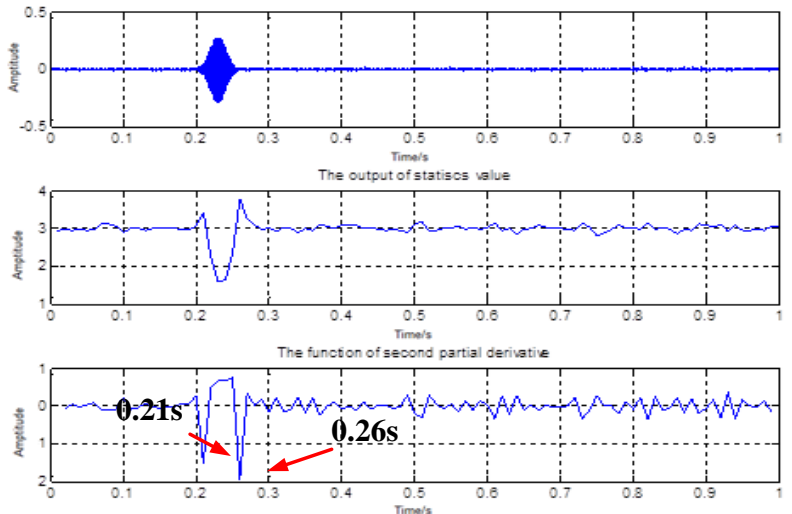

Fig10 The judgment of Gaussian single signal generation and end time

The time-domain waveform of the radiation noise which was derived under the Gaussian working condition was given by Fig10,the kurtosis statistical judgment result was also included.Several points 
cold be inferred from the picture,including the strong power of the transmitted,of course much higher than background,and an obvious character of the Gaussian could be obtained from the waveform,the durable time was pretty short,the signal stands on $6 \mathrm{kHz}$ through power spectrum analysis,the signal belongs to the typical Gaussian amplitude modulation signal.The generation and end time of the impulse signal also could be obtained by comparing crest and trough of the waveform.The starting time was $0.21 \mathrm{~s}$ through the judgment of kurtosis statistical value,ending at $0.26 \mathrm{~s}$, the duration time is $0.05 \mathrm{~s}$,the time interval could include the whole staring and ending time of the impulse signal.

According to the calculating method to the power of transientsignal,the power of the Gaussian modulation signal was:

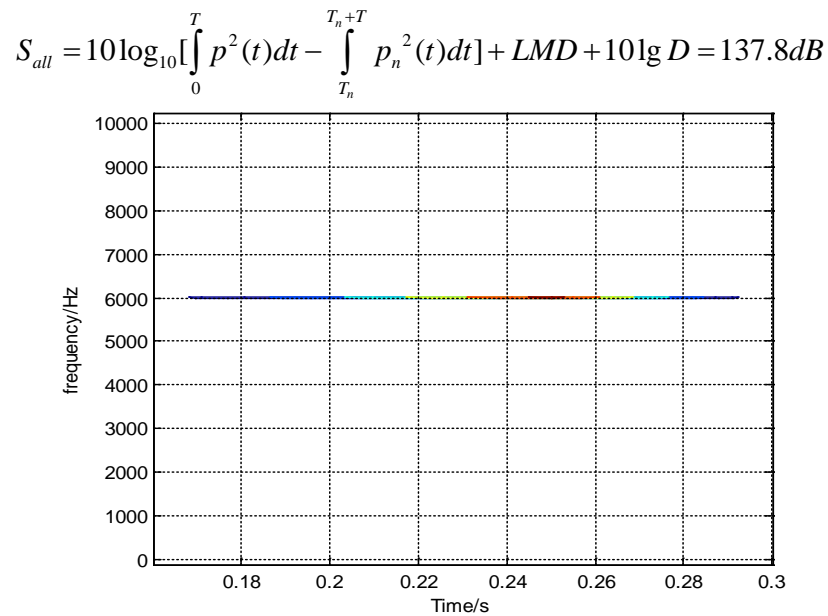

Fig11 Time-frequency analysis result under Gaussian modulation working condition

The time-frequency analysis result of radiation noise of Gaussian modulation signal was given by Fig11,it could be inferred that the center point of the signal stands around 0.25 s, frequency spectrum characteristic performed at $6000 \mathrm{~Hz}$, the generation and end time derived from time-frequency analysis was far more different from the real values, which could illustrate that the precision of Fourier transform is not high enough.

It could be inferred from the time-frequency analysis result that the characteristic signal is a single one stands on $6000 \mathrm{~Hz}$,the signal could be derived from the established signal derivation methods above.

The amplitude of the signal power and waveform before and after the filtering process was given by Fig12,it could be inferred that the power of the filtered characteristic signal was 137.6dB,which only $0.2 \mathrm{~dB}$ difference compared to the original signal,the result proved that the derivation effect was truly ideal.
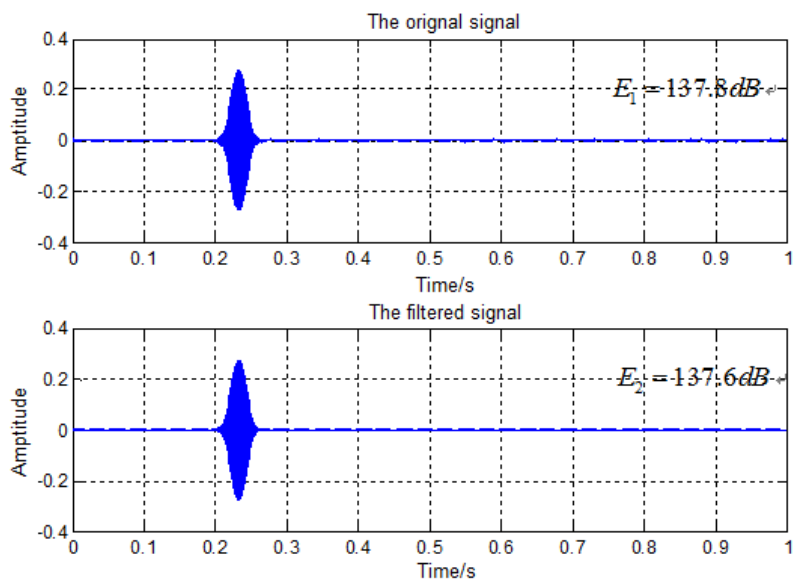

Fig12 Time-domain waveform and magnitude of power before and after filtering

On the basis of the analysis above,the corresponding 1/3oct 6300 frequency band power could be replaced by $6 \mathrm{kHz}$ signal power, the $1 / 3$ oct sound pressure spectrum level could be seen as Fig13,it could be clearly saw from the picture that there was a 6300 summit frequency spectrum existed,which the amplitude was approximately $137.6 \mathrm{~dB}$,the value gets a minimum one to the total sound 
level,which could illustrate the power within $20 \mathrm{~Hz}-50 \mathrm{kHz}$ was totally decided by $6300 \mathrm{~Hz}$,the accuracy of the algorithm could be further verified on this.

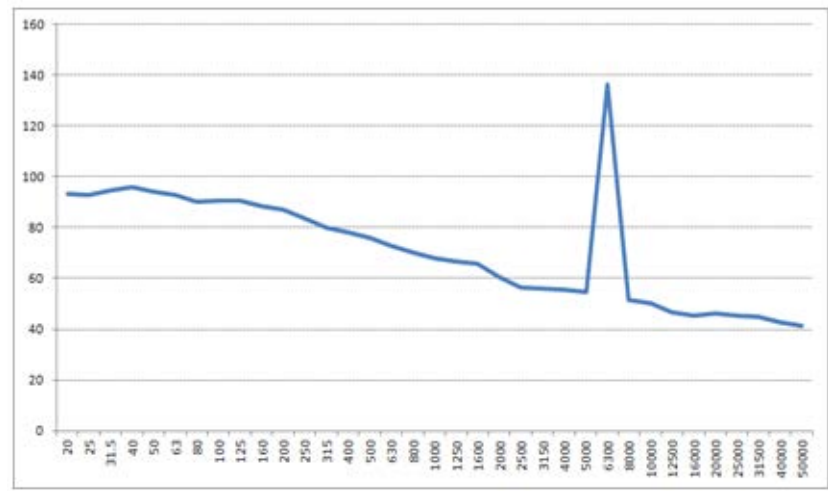

Fig13 Radiation noise 1/3oct sound pressure source spectrum diagram under Gaussian modulation working condition

\section{Error analysis}

The result of the test data analysis are given in the last section, the filtered signal energy is smaller than the original signal, and the difference is about $1 \mathrm{~dB}$. So according to the data processing and analysis process,the main reasons of errors include the marine environment noise, character signal omission, filter passband width and so on.

In this case, the analysis of the factors which may lead to the deviation of the instantaneous energy calculation is carried out according to the case data of explosion sound source.

a. Background factor

In this experiment, the marine background noise is monitored and measured in real time, and the measurement results of background noise during the measurement period are given by Fig14, visible from the figure, the test near the marine background noise is very low.The total sound level $20 \mathrm{~Hz} \sim 50 \mathrm{kHz}$ band basic maintained at around $108 \mathrm{~dB}$, the main characters of the explosion source condition of minimal impact, illustrate the background noise is low enough for the data before and after filtering analysis results have little effect.

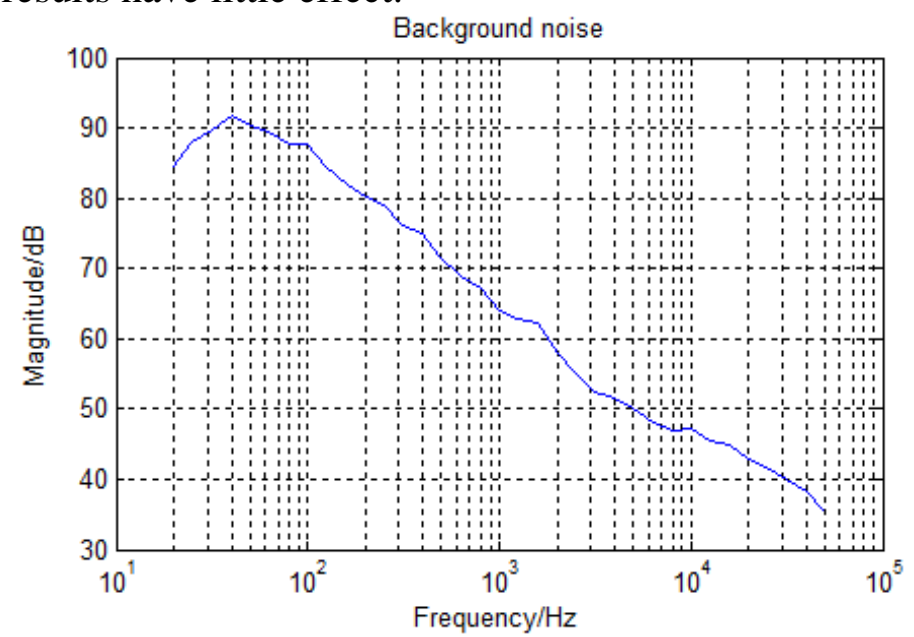

Fig14Measurement results of background noise during transient noise measurements

b. Characteristic information missing

Hypothesis: the energy of the characteristic signal is mainly composed of $P_{1}$ and $P_{2}$,the $P_{1}$ is the main characteristic signal, and the $P_{2}$ is the secondary characteristic signal. In the following, effect of the overall energy of signal is discussed between the main characteristic and the secondary characteristic.

$$
\Delta P=10 \lg \left(10^{\left(P_{1} / 10\right)}+10^{\left(P_{2} / 10\right)}\right)-10 \lg 10^{\left(P_{1} / 10\right)}=10 \lg \left(1+10^{-\left(P_{1}-P_{2}\right) / 10}\right)
$$


The difference between primary characteristic and secondary characteristic affecting the overall energy is shown by fig15, by the diagram shows when $P_{1}-P_{2}=0$, main characteristics and secondary characteristics of the same energy, omitting the secondary characteristics will make the total energy loss of the signal is about $3 \mathrm{~dB}$, and when the difference is $6 \mathrm{~dB}$, secondary features missing for the influence of overall signal energy is less than $1 \mathrm{~dB}$, with the larger the difference and feature a secondary influence on the total energy of the smaller exponential decay trend.

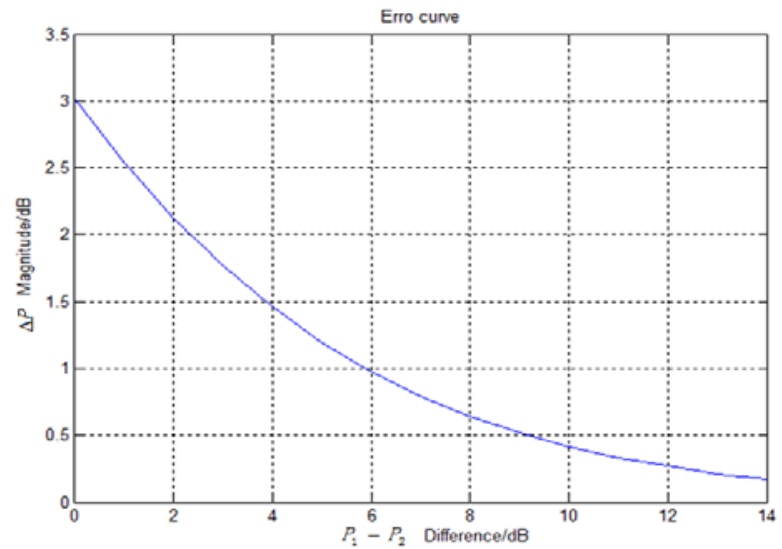

Fig15 Influence of the difference curve on total energy

c. Filter passband width

Set explosion source conditions with main characteristics of $213 \mathrm{~Hz} \sim 726 \mathrm{~Hz}$ as an example, the center frequency of filter is $f_{0}=470 \mathrm{~Hz}$, and passband width is $\Delta f_{1}=514 \mathrm{~Hz}$ and $\Delta f_{2}=480 \mathrm{~Hz}$ respectively. The amplitude frequency response curve is shown in the Fig16, From the figure,as to the filter with passband width of $\Delta f_{1}$, the lower frequency limit can contains the main features $213 \mathrm{~Hz} \sim$ $726 \mathrm{~Hz}$, but for $\Delta f_{2}, 726 \mathrm{~Hz}$ is significantly on the trailing edge of filter's upper frequency ,which will inevitably lead to some energy "leakage" to characteristics of signal .

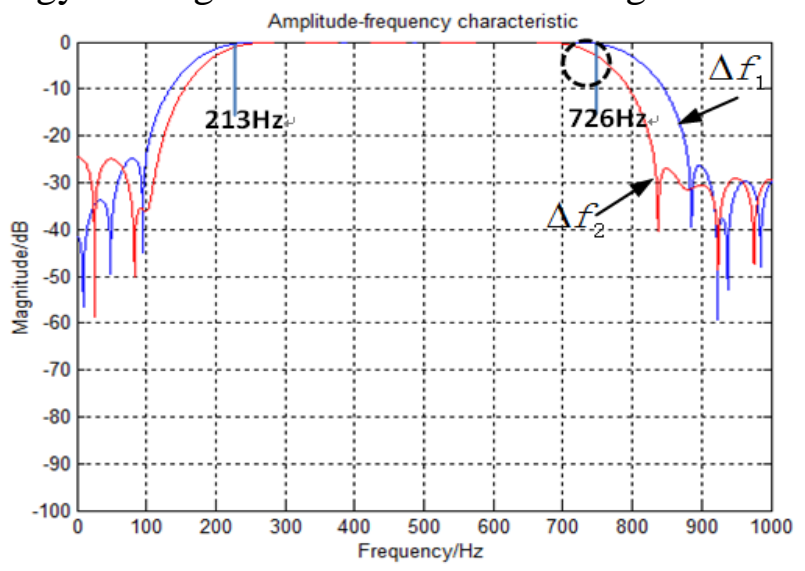

Fig16 Frequency response curve of different passband filter

Waveform and energy of the two filters with different passband are shown by fig17. As you can see from the figure, the calculation result of $\Delta f_{1}$ is higher upon $0.4 \mathrm{~dB}$ than $\Delta f_{2}$. So the filter passband should be arranged as large as possible in order to cover the characteristics of transient signal and to avoid signal energy eigenvalue "leakage". 

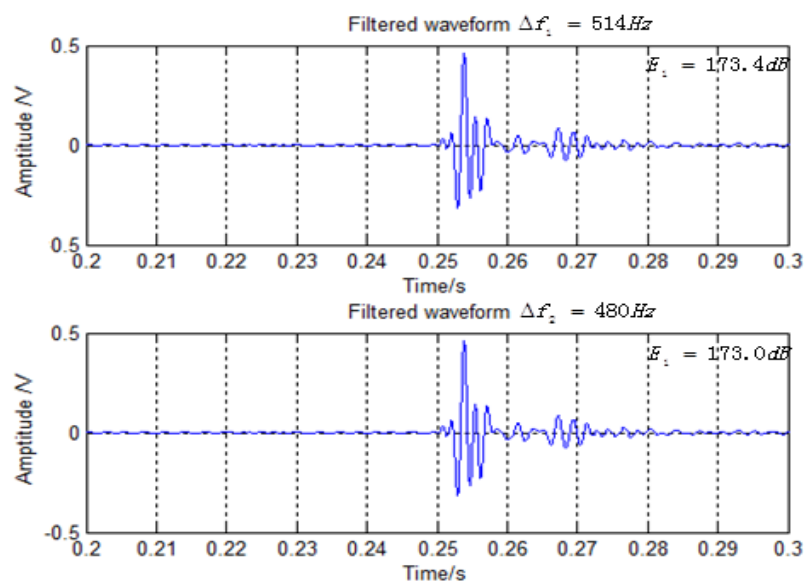

Fig17 Calculation results of different pass band

\section{Summary}

Through different information processing methods towards transit signals.especially the estimation analysis of real data which obtained by the sound source transmit verification experiment on real ship,the below conclusions could be obtained:

(a)The only parameter that could express ship transmit radiation noise underwater is sound energy flux density.

(b)The characteristic frequency spectrum and ampulitude of power of transit signal could be obtained by FIR numberical filter rejector.

(c)A kind of method to derive transit noise characteristic and power calculation is established,the validity and effectiveness of the method is proved through sound source simulation experiment.

(c)The error analysis results show that the bandwidth of the filter is not only necessary to take into account the characteristics of the weak signal, but also as wide as possible, so that finish a full coverage of the characteristic signal.

\section{References}

[1] P.Kirsteins , J.Fay - Suppressing reverberation by multipath separation for improved buried objuect detection. IEEE Oceans, 2001, 236-244P

[2] LV Junjun, WU Guoqing, DU bo. Non-Gaussian under-

water transient signals detection using Power-law detector. Acta Acustica,2004, 29(4).

[3] Wang Yan ,Zou Nan,Fu Jin, Liang Guo-long. Transient Signal Detection Method Based on Partial Instantaneous Energy Density Level[J]. Journal of Electronics \& Information Technology,2013,35(7)

[4]Xiong Xiao-Jun Yin Cheng Zhang Ba-i Lin DING Feng LI Da-Wei. Method Research of Forecasting Oil and Gas Using higher-order Statistics[J]. CHINESE JOURNAL OF GEOPHYSICS, 2004,47(5)

[5]Zhang Guicai Shi Tielin Yang Shuzi. A Method for Extracting Mechanical Faults Features Based on Higher-Order Statistics[J]. J.Huazhong Univ. of Sci. \&Tech, 2004,47(5)

[6]LiGuanfang. The Application of Hilbert-Huang Transform in Transient Signal Processing. A Dissertation for the Degree of M.Eng, 2008

[7]He Chunyan. Analysis and Evaluation for Transient Radiated Noise of Underwater Targets. A Dissertation for the Degree of M.Eng, 2013

[8]Mao Shisong,Cheng Yiming. Probability and mathematical statistics course[M].Higher education press,Beijing,2004

[9]A.Nuttall. Detection performance of power-law processors for random signals of unknown location, structure, extent, and strength. NUWC-NPT Tech. Rep, Sept. 1994. 
[10]You-Ping Li, Wei-Qun Gan, Li Feng, Si-Ming Liu and A. Struminsky. The breakdown of the power-law frequency distributions for the hard X-ray peak count rates of solar flares[J]. Research in Astron. Astrophys. 2013 Vol. 13 No. 12, 1482-1492 